\title{
Inhibition of Angiogenesis and Extracellular Matrix Remodeling: Role of Renin-Angiotensin System Inhibitors in Bevacizumab -induced Hypertension
}

\section{Tianshu Ren}

Shenyang Pharmaceutical University

Hui Jia

General Hospital of Northern Theatre command

Qiong Wu

General Hospital of Northern Theatre command

Yan Zhang

General Hospital of Northern Theatre command

Qun Ma

General Hospital of Northern Theatre command

\section{Dong Yao}

General Hospital of Northern Theatre command

\section{Xudong Gao}

General Hospital of Northern Theatre command

\section{Danni Xie}

General Hospital of Northern Theatre command

\section{Zihua $\mathrm{Xu}$}

General Hospital of Northern Theatre command

\section{Yingshi Zhang}

Shenyang Pharmaceutical University

Chun Qing Zhao ( $\square$ zhaoqingchun1967@163.com )

Shenyang Pharmaceutical University https://orcid.org/0000-0001-9256-9971

\section{Research Article}

Keywords: metastatic colorectal cancer (mCRC), hypertension (HT), RASIs, CCBs, overall survival (OS), progression-free survival (PFS)

Posted Date: August 24th, 2021

DOI: https://doi.org/10.21203/rs.3.rs-830539/v1 
License: (c) (i) This work is licensed under a Creative Commons Attribution 4.0 International License. Read Full License 


\section{Abstract}

Bevacizumab (Bev) is a humanized vascular endothelial growth factor monoclonal antibody that is used with chemotherapeutic drugs for the treatment of metastatic colorectal cancer (mCRC). Bev-induced hypertension ( $\mathrm{HT}$ ) is the most common adverse reaction during clinical practice. However, at present, appropriate antihypertensive agents for Bev-induced HT are unavailable. In this study, retrospective analysis of clinical data from mCRC patients who received renin-angiotensin system inhibitors (RASIs) showed significant survival benefits of overall survival (OS) and progression-free survival (PFS) over patients who received calcium channel blockers (CCBs) and patients who received no antihypertensive drug. An experiment in HCT116 colon cancer cell xenografts in mice confirmed that combined treatment with Bev and lisinopril (Lis), a RASI, synergistically inhibited subcutaneous tumor growth and enhanced the concentration of 5-fluorouracil (5-Fu) in tumor tissues. Our results showed that the addition of Lis did not interfere with the vascular normalization effect promoted by Bev, but also inhibited collagen and hyaluronic acid (HA) deposition and significantly downregulated the expression of TGF- $\beta 1$ and downstream SMAD signaling components which were enhanced by Bev, ultimately remodeling primary ECM components. In conclusion, RASIs might be the optimal choice for the treatment of Bev-induced HT in mCRC patients.

\section{Introduction}

Bevacizumab (Bev) is a humanized vascular endothelial growth factor monoclonal antibody that is used with chemotherapeutic drugs, including 5-fluorouracil (5-Fu) or capecitabine, oxaliplatin or/and irinotecan-based chemotherapy, for the treatment of metastatic colorectal cancer (mCRC)[1-3]. However, Bev-induced hypertension ( $\mathrm{HT}$ ), the most common adverse reaction, has an incidence rate exceeding $30 \%$, which influences the process of anti-tumor treatment and causes cardiovascular events[4, 5],[6].

Guideline recommend when the systolic blood pressure $\geq 165 \mathrm{~mm} \mathrm{Hg}$ or diastolic blood pressure $\geq 100$ $\mathrm{mm} \mathrm{Hg}$, anti-angiogenesis therapy should be combined with anti-hypertensive agents and maintained until the blood pressure reaches to $140 \mathrm{mmHg} / 90 \mathrm{mmHg}$ [7]. For the treatment of essential HT, thiazide diuretics, beta-adrenoceptor antagonists, calcium channel blockers (CCBs), angiotensin converting enzyme inhibitors (ACEls) and angiotensin receptor blockers (ARBs) are widely used[8, 9]. However, at present, appropriate antihypertensive agents for Bev-induced HT are unavailable, because patients undergoing chemotherapy and targeted therapy are excluded from clinical trials[10]. Additionally, given the specific condition of patients with malignant tumors, the choice of antihypertensive agent should not disrupt the antitumor effect of Bev[11, 12]. Instead, it is better to strengthen the antitumor effects of chemotherapies with antiangiogenic drugs [13].

Although Bev was found to affect tumor vascular normalization, it exacerbated more extracellular matrix (ECM) components such as collagen and hyaluronan (HA) deposition[14-18]. ECM constitute an interstitial barrier, which can further increase solid stress and compress tumor vessels, ultimately leading to the insufficient perfusion of chemotherapeutic agents in tumor tissues $[14,19]$. Studies in animal 
models have shown that the deposition of collagen and HA can damage blood vessel function and reduce drug delivery to tumors $[20,21]$. Angiotensin system inhibitors (RASIs), which mainly include ACEls and ARBs, have been confirmed to have therapeutic effects in fibrotic diseases of the lung, myocardium, and kidney [22]. Studies have also reported that RASIs inhibit tumor angiogenesis and reduce the levels of matrix metalloproteinase-2, which involved in degrading ECM and fibrolytic action $[23,24]$.

We hypothesized when treating Bev-induced HT, RASIs could decrease ECM component remodeling induced by Bev, then improve drug delivery in solid tumors. A retrospective analysis of clinical data from $\mathrm{mCRC}$ patients was conducted to investigate which anti-hypertensive agent is more appropriate for Bevinduced HT in mCRC patients. Then, an experiment in HCT116 colon cancer cell xenografts in mice was explored to confirm the combined treatment with Bev and lisinopril (Lis), a RASI, could inhibit angiogenesis and ECM remodeling.

\section{Methods}

\section{Retrospective study design}

A retrospective study of patients diagnosed with $\mathrm{mCRC}$ who were treated with Bev and chemotherapeutics from January 2013 to January 2020 at the General Hospital of Northern Theater Command, China, was performed. The inclusion criteria were the presence of $\mathrm{mCRC}, \geq 1$ site of metastasis with no surgery, treatment with a combination of chemotherapy and Bev for $\geq 2$ cycles, detailed medical records showing blood pressure, and antihypertensive treatment. The exclusion criteria were treatment with chemotherapy regimens that did not meet the inclusion criteria; an insufficient number of chemotherapy and targeted therapy cycles; incomplete medical records with no response evaluation or blood pressure measurement; treatment termination for psychological reasons; drug intolerance or other factors; and surgical resection during chemotherapy and antiangiogenic targeted therapy, local radiofrequency ablation or hepatic artery embolization. Eligible mCRC patients received 5 $\mathrm{mg} / \mathrm{kg}$ Bev every 2 weeks or $7.5 \mathrm{mg} / \mathrm{kg}$ every 3 weeks in combination with 5-Fu or capecitabine \pm oxaliplatin or irinotecan-based chemotherapy. The details of the retrospective study assessments, including patient enrollment, evaluation of treatment response, and hypertension, are shown in the Supplementary Material. The study was conducted according to the Declaration of Helsinki and approved by the Ethics Committee of the General Hospital of Northern Theater Command, China with informed consent waiver (no: Y2020046).

\section{Reagents and antibodies}

Bev injection (100 mg/4 mL, Avastin, Roche Pharma, Switzerland);Lis hydrochloride tablets (10 mg, Zhongfu, China); 5 -Fu injection $(0.25 \mathrm{~g} / \mathrm{mL}$, Xudong, China); $0.9 \%$ sodium chloride injection $(100 \mathrm{~mL}$, Baxter, China);Dulbecco's modified Eagle's medium (DMEM) and fetal bovine serum (FBS) (HyClone, UT, USA); a BCA protein quantification kit (Beyotime Biotechnology, Shanghai, China); an ECL detection kit, anti-TGF- $\beta 1$ and anti-Collagen I (Wanleibio, Shenyang, China); anti-Smad2/3, anti-phospho-Smad2/3 and 
anti-Smad4 (Affinity Biosciences, OH, USA); anti-CD31 and anti-a-SMA (Servicebio, Wuhan, China); antiGAPDH and secondary antibodies for Western blot analysis (Proteintech, Chicago, USA) were used.

\section{Cell lines and cell culture}

Colon cancer cell lines HCT116 were obtained from American Type Culture Collection (ATCC, Manassas, Virginia). The cells were cultured in DMEM supplemented with $1 \%$ penicillin-streptomycin and $10 \%$ FBS in a humidified environment at $37^{\circ} \mathrm{C}$ in a $5 \% \mathrm{CO}_{2}$ atmosphere.

\section{Human colorectal cancer xenograft model}

Female BALB/c-nu mice (4-6 weeks, weight $18 \pm 2 \mathrm{~g}$ ) were purchased from Beijing HFK Bioscience Co., Ltd., (China) and maintained in a special pathogen-free environment. The food and water were available ad libitum. The mice were maintained under a 12-h light / dark cycle at relative humidity $40-60 \%$ and room temperature $24-26^{\circ} \mathrm{C}$. All experimental procedures were carried out in accordance with the guidelines of the Animal Experimental Ethics Committee of the General Hospital of Northern Theater Command. After 5 days of acclimatization to the environment, each mouse was implanted with $0.2 \mathrm{~mL}$ of $5 \times 10^{6} \mathrm{HCT} 116$ cells / $\mathrm{mL}$ by subcutaneous injection into the unilateral axilla to establish one tumor. Tumor length and width were measured by calipers every two days, and the volumes were calculated with the following formula: $V=\left(L \times W^{2}\right) / 2$, where $V=$ the tumor volume $\left(\mathrm{mm}^{3}\right), L=$ the length of the tumor along its major axis $(\mathrm{mm})$, and $\mathrm{W}=$ the width of the tumor along its minor axis $(\mathrm{mm})$. Drug administration began when the tumor volume was $50-70 \mathrm{~mm}^{3}$.

\section{In vivo study design}

The mice were randomly divided into six groups ( $n=12$ in every group): the Con group ( $0.2 \mathrm{~mL}$ PBS, po, per day), Lis group (2.5 mg/kg Lis, po, per day), low-dose Bev group (5 mg/kg Bev, ip, per 3 days), highdose Bev group (10 mg/kg Bev, ip, per 3 days), Lis + low-dose Bev group (2.5 mg/kg Lis, po, per day combined with $5 \mathrm{mg} / \mathrm{kg} \mathrm{Bev}$, ip, per 3 days) and Lis + high-dose Bev group (2.5 mg/kg Lis, po, per day combined with $10 \mathrm{mg} / \mathrm{kg} \mathrm{Bev}$, ip, per 3 days). Dosage of Lis and Bev was calculated based on clinically dose conversion and previous studies $[25,26]$. Tumor volume and mouse weight were measured every 2 days until the end of the experiment on day 12. Tumors were randomly extracted on days 3 and 6 for the quantitative detection of VEGFA ( $n=3$ per time point). On day 12 , the mice were intraperitoneally injected with $20 \mathrm{mg} / \mathrm{kg} 5$-Fu and sacrificed 10 minutes later, after which the tumors were photographed and weighed $(n=6)$. Tumor samples were frozen at $-80^{\circ} \mathrm{C}$ or fixed in paraformaldehyde for further analysis of 5-Fu concentration determination, immunofluorescence, ELISA, Sirius Red staining and western blot.

\section{Determination of the 5-Fu concentration in tumor tissues}

The 5-Fu concentration in tumor tissues was measured with a LC-MS/MS (LC21A, Shimadzu, Japan; Triple Quad ${ }^{\text {TM }} 5500$, SCIEX, USA) system. The 5-Fu reference standard and internal standard, 5bromoutacil (5-Bu), were purchased from Victory Biological Technology Co., Ltd. (Sichuan, China). LC was carried with a Thermo Scientific ${ }^{\mathrm{TM}}$ Hypersil GOLD ${ }^{\mathrm{TM}}$ column $(5 \mu \mathrm{m}, 4.6 \mathrm{~mm} \times 150 \mathrm{~mm})$ and the following conditions: mobile phase: methanol-formic acid-deionized water (50: 0.1: 49.9), isocratic elution mode,10- 
minute elution time, $0.3 \mathrm{~mL} / \mathrm{min}$ flow rate, $35^{\circ} \mathrm{C}$ column temperature, and a $5-\mu \mathrm{L}$ injection volume. The mass spectrometer was equipped with an electro spray ionization (ESI) probe. The multiple reaction monitoring (MRM) m/z transitions monitored were 128.9-42.1 (CE - $33 \mathrm{~V}$ ) for 5-Fu and 188.8-42.1 (CE -37 V) for 5-Bu.

\section{Immunofluorescence staining and analyses}

The paraformaldehyde-fixed tumor tissues were embedded in paraffin and cut into sections $(5 \mu \mathrm{m})$ with a slicer (KD-2508, Zhejiang Jinhua Kedi Instrumental Equipment CO., LTD, China). The paraffin sections underwent dewaxing, hydration, antigen retrieval, and serum blocking and were then incubated with antiCD31 antibody (1:100) and anti-a-SMA antibody (1:500) at $4^{\circ} \mathrm{C}$ overnight. Cy3 goat anti-rabbit IgG (1:300) and 488 goat anti-mouse IgG (1:400) were then added. After the nuclei were counterstained with DAPI and auto fluorescence had been quenched, the stained tissues were observed and imaged by inverted fluorescence microscopy (Olympus BX53). Morphological observation and quantitative analysis were conducted with Image J software (version 1.49, National Institutes of Health, Bethesda, MD, USA). Nine optical fields of CD31+hot spots per tumor section were selected in high-power vision $(\times 200)$. The MVD was calculated by counting the number of individual CD31-positive luminal structures in each field. The VMI was calculated by determining the ratio of areas doubly positive for CD31 and a-SMA in each field according to the previous studies [27-29].

\section{VEGFA and HA ELISAs of tumor tissues}

Tumor tissues were weighed, cut into small pieces, homogenized in cold PBS ( $\mathrm{pH} 7.4)$ and centrifuged at $3,000 \times g$ for 20 minutes at $4^{\circ} \mathrm{C}$ to obtain supernatant samples. Protein expression in the tumor tissues was measured with ELISA kits for VEGFA (Cloud-Clone Corp, China) and HA (Mskbio, China) according to the manufacturers' instructions.

\section{Evaluation of collagen in tumor tissues}

Collagen deposition in tumor tissues was investigated by Sirius Red staining. Paraffin sections were dewaxed and hydrated via graded ethanol $(70 \%, 85 \%, 95 \%$, and $100 \%, \mathrm{v} / \mathrm{v})$ before being stained with Weigert's iron hematoxylin solution for 10-20 minutes. Following differentiation with acidic differentiation solution and washing with distilled water, the sections were stained with Sirius red staining solution for 1 hour, gently rinsed, dehydrated with ethanol, cleared with xylene, and gradually mounted with neutral gum. Finally, the slices were imaged with an ordinary optical microscope and a polarized optical microscope.

\section{Protein extraction and Western blot analysis}

Tumor tissues were lysed with RIPA lysis buffer (Beyotime Biotechnology, Shanghai, China). Protein concentrations were detected by BCA protein assay kits (Beyotime Biotechnology, Shanghai, China). Proteins ( $50 \mathrm{\mu g}$ / lane) were electrophoretically separated on 12\% SDS-PAGE gels and transferred to PVDF membranes. The membranes were immuneblotted with specific primary antibodies and then incubated with HRP-conjugated secondary antibody. HRP was detected with an ECL system (Beyotime 
Biotechnology, Shanghai, China). The resultant bands were imaged by a ChemiDoc Touch Imaging System (Bio-Rad Laboratories, Inc., USA) and quantified by Image J software.

\section{Statistical analyses}

Datasets were analyzed by Student's t-test or ANOVA, and the results are shown as the mean \pm SEM. Statistical significance was defined at $P<0.05$. For the retrospective analysis, the Pearson's chi-square test or Fisher's exact test was used to assess differences between the groups. progression-free survival (PFS) and overall survival (OS) curves were estimated using the Kaplan-Meier method, while the associations between survival time and predictor variables were statistically investigated using the Cox proportional hazards regression model. All calculations were performed using IBM SPSS Statistics 25.0 (SPSS, Inc., Chicago, IL, USA) and GraphPad Prism 7.0 (GraphPad Software, Inc., San Diego, CA, USA).

\section{Results}

\section{Patient characteristics in the retrospective study}

A retrospective analysis of 94 patients with $\mathrm{mCRC}$ was conducted to investigate the optimal choice of anti-hypertensive agent for the treatment of Bev-induced $\mathrm{HT}$. Eligible patients received Bev in combination with 5-Fu or capecitabine \pm oxaliplatin or irinotecan-based chemotherapy were included: 63 males $(67.02 \%)$ and 31 females (32.98\%). The median age was 58 years. For forty-four patients $(46.81 \%)$, the primary lesion was in the rectum, and for fifty patients (53.19\%), it was in the colon. Fifty-six patients (59.57\%) underwent surgical resection before chemotherapy and Bev therapy. Twenty-five patients (26.60\%) had a history of HT controlled with antihypertensive drugs.

The patients were divided into a Bev-induced HT (Bev-HT) group $(n=41)$ and a non-HT group $(n=53)$ based on blood pressure measurements and assessment criteria (Supplementary Table 1). There were no differences between the two groups in age, sex, Karnofsky Performance Status (KPS), pathological tissue genotype, primary lesion location, or comorbidities including hypertension and coronary heart disease. The Bev-HT group included more patients with diabetes $(22.00 \% v s .13 .2 \%, P=0.03)$.

In the Bev-HT group, both systolic blood pressure (SBP) and diastolic blood pressure (DBP) increased significantly after Bev treatment (SBP, $125.2 \pm 11.54 \mathrm{mmHg} v$ s. $154.6 \pm 12.89 \mathrm{mmHg}$; DBP, $79.15 \pm 7.02$ $\mathrm{mmHg} v$ s. $94.88 \pm 8.34 \mathrm{mmHg}, P<0.001)$. According to the Common Terminology Criteria for Adverse Events version 5.0 (CTCAE v5.0) [7], 27 patients in the Bev-HT group were classified as developing grade $2 \mathrm{HT}$, and 14 patients developed grade $3 \mathrm{HT}$, but no patients developed grade $\geq 4 \mathrm{HT}$. PFS and OS were found to significantly differ between the Bev-HT group and non-HT group [median PFS 10.5 vs. 5.5 mo, hazard ratio (HR) 0.3049, 95\% confidence interval (Cl) 0.1809-0.514, $P<0.0001$; median OS 16.0 vs. 11.0 mo, HR 0.3798, 95\% Cl0.2161-0.6676, $P=0.0008$ ] (Fig. 1A-B).

\section{Adjunctive treatment with RASIs improves survival in patients with MCRC}


Then, subgroups according to different antihypertensive treatment were divided for the further analysis. Compared with patients who received CCBs $(n=19)$, patients who received RASIs $(n=18)$ had a longer PFS and OS (median PFS 13.00 vs. 7.0 mo, HR 0.3961, 95\% Cl0.1753-0.8951, $P=0.0260$; median OS 18.00 vs. $11.00 \mathrm{mo}, \mathrm{HR} 0.251495 \% \mathrm{Cl} 0.1066-0.5929, P=0.0016)$. Moreover, we observed a significant larger improvement in PFS and OS in the patients who received RASIs than the patients who did not receive any antihypertensive drugs $(\mathrm{n}=57)$ (median PFS, 13.00 vs. $7.00 \mathrm{mo}, \mathrm{HR} 0.2870,95 \% \mathrm{Cl} 0.1579-$ $0.5214, P<0.0001$; median OS 18.00 vs. 13.00 mo; HR 0.3708, 95\% Cl0.1992-0.6902, $P=0.0018$ )

(Fig. 1C-D). The result of multivariate regression analysis showed that RASI use is a significant factor for the improvement of PFS and OS. Moreover, irinotecan-based chemotherapy and 7-12 cycles of Bev treatment were found to be independently predictive of improved OS, while diabetes and $\geq 3$ metastatic sites were risk factors for poor prognosis in the patients (Fig. 1E). The results of this retrospective study indicated that when treated Bev-HT, RASIs improves survival in patients with mCRC effectively.

\section{Bev and Lis synergistically inhibited the growth of HCT116 xenograft tumors in mice}

A human HCT116 colon cancer cell xenograft mouse model was established, and the inhibitory effect on tumor volume of the angiotensin-converting enzyme inhibitor (ACEI) Lis and Bev on subcutaneous tumors was investigated. The mice were randomly divided into six groups and treated according to doseconversion between mice and humans and previous studies[25, 26]: the control group (Con, 200 $\mu \mathrm{L}$ PBS, po, per day), Lis group (2.5 mg/kg Lis, po, per day), low-dose Bev group [5 mg/kg Bev, intraperitoneal (ip), per 3 days], high-dose Bev group (10 mg/kg Bev, ip, per 3 days), Lis combined with low-dose Bev group ( $2.5 \mathrm{mg} / \mathrm{kg}$ Lis, po, per day; $5 \mathrm{mg} / \mathrm{kg}$ Bev, ip, per 3 days) and Lis combined with high-dose Bev group (2.5 $\mathrm{mg} / \mathrm{kg}$ Lis, po, per day; $10 \mathrm{mg} / \mathrm{kg}$ Bev, ip, per 3 days).

A schematic of the study design is shown in Fig. 2A. After treatment with Bev monotherapy $(5 \mathrm{mg} / \mathrm{kg}$ and $10 \mathrm{mg} / \mathrm{kg}$ ), tumors resolved rapidly and lastingly, which was significant and dose-dependent (Fig. 2C-D). Although Lis monotherapy tended to slow tumor growth compared with the Con condition, this difference was not statistically significant. Notably, combination treatment with Bev and Lis significantly enhanced the inhibition of tumor growth induced by Bev monotherapy (Fig. 2B-D). According to the formula of drug combination $\mathrm{q}=\mathrm{E}(\mathrm{A}+\mathrm{B}) /(\mathrm{EA}+\mathrm{EB}-\mathrm{EA} \times \mathrm{EB})[30,31]$, Bev and Lis were determined to synergistically suppress tumor growth $(q=1.41)$. The weight of the mice in each group decreased throughout the 12 days of the experiment, with no significant difference among the groups (Fig. 2E). Thus, the results of these in vivo experiments indicated that the synergistic therapeutic effects of Bev and Lis are better than the effect of single-agent antitumor therapy.

\section{Bev and Lis synergistically increased5-fluorouracil (5-Fu) delivery to tumor tissues}

To study the penetration of chemotherapy drugs, we then determined the concentration of 5-Fu in xenograft tumors by liquid chromatography tandem mass spectrometry (LC-MS/MS). Bev monotherapy 
(10 mg/kg) and the addition of Lis $(2.5 \mathrm{mg} / \mathrm{kg})$ significantly increased the concentration of 5-Fu in tumor tissues compared with that of the Con group (Fig. 3A). In particular, tumor tissues in the combination therapy groups showed higher 5-Fu concentrations than those in the Bev monotherapy groups. The results indicated that Bev and Lis synergistically increased 5-Fu delivery to tumor tissues

\section{Antiangiogenic effects of Bev and Lis}

VEGFA, the primary member of the VEGF family, directly functions in the proliferation and permeability of endothelial cells and induces physiological angiogenesis in $\mathrm{MCRC}$ [32]. Therefore, to study the angiogenesis effects of Bev and Lis, we investigated the levels of VEGFA in the xenograft tumor tissues of mice in the different treatment groups at different time points. After 3 and 6 days of treatment, compared with the Con group and Lis monotherapy group, the $10 \mathrm{mg} / \mathrm{kg}$ Bev monotherapy group and the two Lis and Bev combination groups inhibited VEGFA levels significantly (Fig. 3B). Moreover, as the treatment was prolonged to 12 days, the two combination groups showed dose-dependent significant differences in decreasing VEGFA compared with the Bev monotherapy groups. The results indicated that combined treatment with Bev and Lis could synergistically inhibit VEGFA, which inhibit angiogenesis (Fig. 3B).

Anti-angiogenic therapies, which 'normalize' the abnormal blood vessels in tumors, have been proven to improve the delivery and effectiveness of chemotherapeutics[33]. The synergistic effects of Bev and Lis on tumor blood vessel normalization were investigated by CD31 and a-SMA immunofluorescence double staining (Fig. 3C). Compared with $5 \mathrm{mg} / \mathrm{kg} \mathrm{Bev,} 2.5 \mathrm{mg} / \mathrm{kg}$ Lis did not contribute to the effects of Bev on MVD or VMI in tumor tissues, which confirmed that Lis did not disrupt the ability of Bev to normalize the vasculature (Fig. 3D-E). a-SMA is the gold standard marker of fibroblast activation[34]. Compared with the Con group and the $5 \mathrm{mg} / \mathrm{kg}$ Bev group, the Lis group showed a significant decrease in fibroblasts, as demonstrated by a-SMA staining $(P=0.0028)$. These findings suggest that Lis prevents the fibrotic infiltration of tumor tissues (Fig. 3F).

\section{Lis reduces extracellular matrix (ECM) component remodeling by Bev}

Collagens, fibronectins and proteoglycans, including $\mathrm{HA}$, are the primary ECM components in colon cancer tissue, and the ECM level increases during the malignant pathological process [35-37]. Collagen I expression after Bev and Lis intervention was investigated by Sirius Red staining and Western blot assays. The results indicated that compared with the control treatment, Bev treatment significantly increased collagen fiber deposition and higher collagen I protein expression, while Lis treatment decreased these parameters (Fig. 4A-E). The results of enzyme-linked immunosorbent assay (ELISA) showed that compared with the Con condition, Lis monotherapy significantly decreased the expression level of HA $(P=0.0214)$. Furthermore, compared with Bev treatment, Lis treatment significantly reduced the HA level in tumor tissues $(P=0.0009)(F i g .4 F)$. The combination therapy of Bev and Lis significantly decreased the collagen and HA level compared with Bev monotherapy. These results suggest that Lis could reverse ECM component remodeling induced by Bev. 


\section{Lis inhibits ECM remodeling induced by Bev by downregulating the TGF- $\beta 1 /$ SMAD signaling pathway}

ECM remodeling results in interstitial barriers and induces insufficient perfusion, which can be exacerbated by antiangiogenic treatment $[38,39]$. To clarify the underlying mechanisms by which Lis reversed Bev-induced ECM remodeling, we further evaluated the protein expression of TGF- $\beta 1$, an ECMrelated signaling pathway marker[40]. Compared with that in the Con group, the expression level of TGF$\beta 1$ was significantly decreased in the Lis group; moreover, Lis inhibited the Bev-induced increase in TGF$\beta 1$ expression (Fig. 4G-H). TGF- $\beta 1$ promotes tissue fibrosis, which triggers downstream SMAD signaling pathways, and participates in ECM synthesis and deposition during biological processes [41]. Compared with the Con group and the Bev group, the Lis group showed significantly decreased total-Smad2/3, phosphorylated-Smad2/3, and Smad4 protein expression (Fig. 4I-K). These findings indicate that Lis might inhibit ECM remodeling induced by Bev by downregulating the expression of components in the TGF- $\beta 1 /$ SMAD2/3 signaling pathway.

\section{Discussion}

To explore the appropriate antihypertensive agents for Bev-induced HT, we first conducted a retrospective analysis to explore the survival benefits of different antihypertensive drugs on patients with mCRC who were treated with Bev and standard chemotherapy. Compared with those who received CCBs, patients who received ACEls or ARBs had a longer PFS and OS. Then, we performed an in vivo study of HCT116 colon cancer xenografts in mice and confirmed that Bev and Lis synergistically inhibited tumor growth and increased the 5-Fu concentration in tumor tissues. The findings indicate that RASls might be the optimal choice for Bev-induced HT in the treatment of mCRC.

Our study demonstrated that Lis monotherapy tended to inhibit tumor growth and VEGFA expression, but compared with the control group, the difference was not statistically significant. Moreover, the addition of Lis did not contribute to effects of Bev on MVD or the VMI, which indicated that Lis did not directly promote the penetration of 5-Fu, a chemotherapeutic drug, by vascular normalization. Instead, Lis might have cooperated with Bev through another mechanism to increase the concentration of 5-Fu in tumors. Previous research has suggested the vascular normalization and solid stress alleviation treatments are promising strategies to improve tumor perfusion and delivery of drugs[42]. Although Bev improved tumor vascular normalization, it showed a significant increase in fibroblasts of tumor tissues, as demonstrated by a-SMA staining, and exacerbated collagen and HA deposition, which could damage blood vessel function and reduce drug delivery to tumors. Our results suggest Lis could reverse ECM component enhanced by Bev.

Subsequent experiments suggested that Lis inhibits TGF- $\beta 1$, a multifunctional cytokine associated with ECM component remodeling, tumor angiogenesis, invasion, and metastasis[43]. Furthermore, TGF- $\beta 1$ levels were previously shown to be significantly increased and associated with poor clinical prognosis in colorectal cancer $[44,45]$. TGF- $\beta$ subfamily signaling begins with TGF- $\beta$ ligand activation and binding to 
Ser/Thr kinase receptors, which in turn causes phosphorylation of the downstream target Smad2/3. Phosphorylated Smad2/3 forms a heterodimeric complex with Smad4 that then translocates to the nucleus [46]. Our results showed that the expression of TGF- $\beta 1$ and downstream SMAD signaling components was enhanced by Bev, ultimately remodeling primary ECM components, whereas Lis inhibited these effects.

This study has some limitations. Due to the limited number of patients in the retrospective study, we did not establish subgroups to further evaluate whether there were differences in survival benefits between patients treated with ACEls and ARBs. Studies indicated that angiotensin-II-receptor-1 (AT1) and angiotensin-II-receptor-2 (AT2) signaling have opposite effects on tumor fibrosis; thus, ACEls reduced collagen I and hyaluronan levels to a lesser extent than ARBs $[47,48]$. However, Rahbari et al. demonstrated that AT1 deletion did not affect HA expression, and the abnormal deposition of ECM observed after anti-VEGF therapy was independent of the AT1 pathway[14]. HT of all grades has been observed in up to $36 \%$ of patients treated with Bev, and the reported incidence of high-grade HT ranges from $1.8-22 \%$, with up to $1 \%$ of events being grade 4 in clinical trials [49]. Considering the intensity of its antihypertensive effect and long-term cardioprotection, ACEl treatment is more likely to reduce stroke, nonfatal myocardial infarction, and cardiovascular and total mortality in high-risk patients than ARB treatment[50].

In summary, mCRC patients who received RASIs were found to have a significantly better survival prognosis than the patients who received CCBs. The combination of Bev and Lis inhibited angiogenesis and ECM component deposition, which promoted 5-Fu perfusion into the tumor. Thus, RASIs might be the optimal choice for Bev-induced HT.

\section{Declarations}

\section{Acknowledgments}

This study was supported by a Liaoning Science and Technology Research Project (no. 2020-BS-125).

\section{Conflict of interest}

The authors declare that there are no conflicts of interest related to this study.

\section{Authors' contributions}

RT, ZYS and ZQ conceived of and designed the research.

$R T, J H, W Q, M Q, Y D, X D$ and $X Z$ performed the research.

RT, ZY and JH analyzed the data and wrote the manuscript.

$\mathrm{JH}, \mathrm{GX}, \mathrm{ZYS}$ and ZQ reviewed the manuscript. 
All authors have made an intellectual contribution to the manuscript and approved its submission.

\section{References}

1. Van Cutsem E, Cervantes A, Adam R, Sobrero A, Van Krieken JH, Aderka D et al (2016) ESMO consensus guidelines for the management of patients with metastatic colorectal cancer. Ann Oncol 27(8):1386-1422. doi:10.1093/annonc/mdw235

2. Watanabe T, Muro K, Ajioka Y, Hashiguchi Y, Ito Y, Saito Y et al (2018) Japanese Society for Cancer of the Colon and Rectum (JSCCR) guidelines 2016 for the treatment of colorectal cancer. Int J Clin Oncol 23(1):1-34. doi:10.1007/s10147-017-1101-6

3. Messersmith WA (2017) Systemic Management of Colorectal Cancer. J Natl Compr Canc Netw 15(5s):699-702. doi:10.6004/jnccn.2017.0077

4. El-Kenawi AE, El-Remessy AB (2013) Angiogenesis inhibitors in cancer therapy: mechanistic perspective on classification and treatment rationales. $\mathrm{Br} J$ Pharmacol 170(4):712-729. doi:10.1111/bph.12344

5. Tampellini M, Sonetto C, Scagliotti GV (2016) Novel anti-angiogenic therapeutic strategies in colorectal cancer. Expert Opin Investig Drugs 25(5):507-520. doi:10.1517/13543784.2016.1161754

6. Li M, Kroetz DL (2018) Bevacizumab-induced hypertension: Clinical presentation and molecular understanding. Pharmacol Ther 182:152-160. doi:10.1016/j.pharmthera.2017.08.012

7. Institute NC (2017) Common Terminology Criteria for Adverse Events Version 5.0 published November 27, Retrieved from http://ctep.cancer.gov/reporting/ctc.html 2017

8. Cuspidi C, Tadic M, Grassi G, Mancia G (2018) Treatment of hypertension: The ESH/ESC guidelines recommendations. Pharmacol Res 128:315-321. doi:10.1016/j.phrs.2017.10.003

9. Whelton PK, Carey RM, Aronow WS, Casey DE Jr, Collins KJ, Dennison Himmelfarb C et al 2017 ACC/AHA/AAPA/ABC/ACPM/AGS/APhA/ASH/ASPC/NMA/PCNA Guideline for the Prevention, Detection, Evaluation, and Management of High Blood Pressure in Adults: A Report of the American College of Cardiology/American Heart Association Task Force on Clinical Practice Guidelines. Hypertension 2018;71(6):e13-e115.doi:10.1161/hyp.0000000000000065

10. Chobanian AV, Bakris GL, Black HR, Cushman WC, Green LA, Izzo JL Jr et al (2003) The Seventh Report of the Joint National Committee on Prevention, Detection, Evaluation, and Treatment of High Blood Pressure: the JNC 7 report. Jama 289(19):2560-2572. doi:10.1001/jama.289.19.2560

11. Zamorano JL, Lancellotti P, Muñoz DR, Aboyans V, Asteggiano R, Galderisi M et al (2016) 2016 ESC Position Paper on cancer treatments and cardiovascular toxicity developed under the auspices of the ESC Committee for Practice Guidelines. Kardiol Pol 74(11):1193-1233. doi:10.5603/kp.2016.0156

12. Tarantini L, Gulizia MM, Di Lenarda A, Maurea N, Giuseppe Abrignani M, Bisceglia I et al (2017) ANMCO/AIOM/AICO Consensus Document on clinical and management pathways of cardiooncology: executive summary. Eur Heart J Suppl 19(Suppl D):D370-Dd379.

doi:10.1093/eurheartj/sux019

Page 12/21 
13. Ferroni P, Della-Morte D, Palmirotta R, Rundek T, Guadagni F, Roselli M (2012) Angiogenesis and hypertension: the dual role of anti-hypertensive and anti-angiogenic therapies. Curr Vasc Pharmacol 10(4):479-493. doi:10.2174/157016112800812836

14. Rahbari NN, Kedrin D, Incio J, Liu H, Ho WW, Nia HT et al (2016) Anti-VEGF therapy induces ECM remodeling and mechanical barriers to therapy in colorectal cancer liver metastases. Sci Transl Med 8(360):360ra135. doi:10.1126/scitranslmed.aaf5219

15. Jain RK (2014) Antiangiogenesis strategies revisited: from starving tumors to alleviating hypoxia. Cancer Cell 26(5):605-622. doi:10.1016/j.ccell.2014.10.006

16. Coulson-Thomas VJ, Coulson-Thomas YM, Gesteira TF, de Paula CA, Mader AM, Waisberg J et al (2011) Colorectal cancer desmoplastic reaction up-regulates collagen synthesis and restricts cancer cell invasion. Cell Tissue Res 346(2):223-236. doi:10.1007/s00441-011-1254-y

17. Leight JL, Wozniak MA, Chen S, Lynch ML, Chen CS (2012) Matrix rigidity regulates a switch between TGF- $\beta 1$-induced apoptosis and epithelial-mesenchymal transition. Mol Biol Cell 23(5):781-791. doi:10.1091/mbc.E11-06-0537

18. Chen Y, Wang W, Jiang B, Yao L, Xia F, Li X (2020) Integrating Tumor Stroma Biomarkers With Clinical Indicators for Colon Cancer Survival Stratification. Front Med (Lausanne) 7:584747. doi:10.3389/fmed.2020.584747

19. Aguilera KY, Rivera LB, Hur H, Carbon JG, Toombs JE, Goldstein CD et al (2014) Collagen signaling enhances tumor progression after anti-VEGF therapy in a murine model of pancreatic ductal adenocarcinoma. Cancer Res 74(4):1032-1044. doi:10.1158/0008-5472.can-13-2800

20. Carmeliet P, Jain RK (2011) Molecular mechanisms and clinical applications of angiogenesis. Nature 473(7347):298-307. doi:10.1038/nature10144

21. Welti J, Loges S, Dimmeler S, Carmeliet P (2013) Recent molecular discoveries in angiogenesis and antiangiogenic therapies in cancer. J Clin Invest 123(8):3190-3200. doi:10.1172/jci70212

22. Yang T, Chen YY, Liu JR, Zhao H, Vaziri ND, Guo Y et al (2019) Natural products against reninangiotensin system for antifibrosis therapy. Eur J Med Chem 179:623-633.

doi:10.1016/j.ejmech.2019.06.091

23. Volpert OV, Ward WF, Lingen MW, Chesler L, Solt DB, Johnson MD et al (1996) Captopril inhibits angiogenesis and slows the growth of experimental tumors in rats. J Clin Invest 98(3):671-679. doi:10.1172/jci118838

24. Saber S, Mahmoud AAA, Goda R, Helal NS, El-Ahwany E, Abdelghany RH (2018) Perindopril, fosinopril and losartan inhibited the progression of diethylnitrosamine-induced hepatocellular carcinoma in mice via the inactivation of nuclear transcription factor kappa-B. Toxicol Lett 295:3240. doi:10.1016/j.toxlet.2018.05.036

25. von Baumgarten L, Brucker D, Tirniceru A, Kienast Y, Grau S, Burgold S et al (2011) Bevacizumab has differential and dose-dependent effects on glioma blood vessels and tumor cells. Clin Cancer Res 17(19):6192-6205. doi:10.1158/1078-0432.ccr-10-1868 
26. Gerber HP, Ferrara N (2005) Pharmacology and pharmacodynamics of bevacizumab as monotherapy or in combination with cytotoxic therapy in preclinical studies. Cancer Res 65(3):671680

27. Eberhard A, Kahlert S, Goede V, Hemmerlein B, Plate KH, Augustin HG (2000) Heterogeneity of angiogenesis and blood vessel maturation in human tumors: implications for antiangiogenic tumor therapies. Cancer Res 60(5):1388-1393

28. Alarcon-Martinez L, Yilmaz-Ozcan S, Yemisci M. Capillary pericytes express a-smooth muscle actin, which requires prevention of filamentous-actin depolymerization for detection. 2018;7.doi:10.7554/eLife.34861

29. Yonenaga $Y$, Mori A, Onodera H, Yasuda S, Oe H, Fujimoto A et al (2005) Absence of smooth muscle actin-positive pericyte coverage of tumor vessels correlates with hematogenous metastasis and prognosis of colorectal cancer patients. Oncology 69(2):159-166. doi:10.1159/000087840

30. Yao R, Zheng J, Zheng W, Gong Y, Liu W, Xing R (2014) VX680 suppresses the growth of HepG2 cells and enhances the chemosensitivity to cisplatin. Oncol Lett 7(1):121-124. doi:10.3892/ol.2013.1648

31. ZJ J. Addition in drug combination. Zhongguo Yao Li Xue Bao 1980;1

32. Slattery ML, Lundgreen A, Wolff RK. VEGFA (2014) FLT1, KDR and colorectal cancer: assessment of disease risk, tumor molecular phenotype, and survival. Mol Carcinog 53(Suppl 1):E140-E150. doi:10.1002/mc.22058

33. Jain RK (2005) Normalization of tumor vasculature: an emerging concept in antiangiogenic therapy. Science 307(5706):58-62. doi:10.1126/science.1104819

34. Vera R, Alonso V, Gállego J, González E, Guillén-Ponce C, Pericay C et al (2015) Current controversies in the management of metastatic colorectal cancer. Cancer Chemother Pharmacol 76(4):659-677. doi:10.1007/s00280-015-2808-6

35. Miura S, Kodaira S, Hosoda Y (1993) Immunohistologic analysis of the extracellular matrix components of the fibrous stroma of human colon cancer. J Surg Oncol 53(1):36-42. doi:10.1002/jso.2930530111

36. Jojovic M, Delpech B, Prehm P, Schumacher U (2002) Expression of hyaluronate and hyaluronate synthase in human primary tumours and their metastases in scid mice. Cancer Lett 188(1-2):181189. doi:10.1016/s0304-3835(02)00037-x

37. Laurich C, Wheeler MA, lida J, Neudauer CL, McCarthy JB, Bullard KM (2004) Hyaluronan mediates adhesion of metastatic colon carcinoma cells. J Surg Res 122(1):70 -70 4. doi:10.1016/j.jss.2004.05.018

38. Ellis LM, Hicklin DJ (2008) VEGF-targeted therapy: mechanisms of anti-tumour activity. Nat Rev Cancer 8(8):579-591. doi:10.1038/nrc2403

39. Rapisarda A, Shoemaker RH, Melillo G (2009) Antiangiogenic agents and HIF-1 inhibitors meet at the crossroads. Cell Cycle 8(24):4040-4043. doi:10.4161/cc.8.24.10145

40. Meng XM, Nikolic-Paterson DJ, Lan HY (2016) TGF- $\beta$ : the master regulator of fibrosis. Nat Rev Nephrol 12(6):325-338. doi:10.1038/nrneph.2016.48 
41. Hu HH, Chen DQ, Wang YN, Feng YL, Cao G, Vaziri ND et al (2018) New insights into TGF- $\beta /$ Smad signaling in tissue fibrosis. Chem Biol Interact 292:76-83. doi:10.1016/j.cbi.2018.07.008

42. Stylianopoulosa T, Jainb RK (2013) Combining two strategies to improve perfusion and drug delivery in solid tumors. PNAS 110(46):18632-18637

43. Ding A, Bian YY, Zhang ZH (2020) SP1/TGF- $\beta 1 / S M A D 2$ pathway is involved in angiogenesis during osteogenesis. Mol Med Rep 21(3):1581-1589. doi:10.3892/mmr.2020.10965

44. Lampropoulos P, Zizi-Sermpetzoglou A, Rizos S, Kostakis A, Nikiteas N, Papavassiliou AG (2012) TGF-beta signalling in colon carcinogenesis. Cancer Lett 314(1):1-7.

doi:10.1016/j.canlet.2011.09.041

45. Tsushima H, Ito N, Tamura S, Matsuda Y, Inada M, Yabuuchi I et al (2001) Circulating transforming growth factor beta 1 as a predictor of liver metastasis after resection in colorectal cancer. Clin Cancer Res 7(5):1258-1262

46. Zhao M, Mishra L, Deng CX (2018) The role of TGF-beta/SMAD4 signaling in cancer. Int J Biol Sci 14(2):111-123. doi:10.7150/ijbs.23230

47. Zhao Y, Cao J, Melamed A, Worley M, Gockley A, Jones D et al (2019) Losartan treatment enhances chemotherapy efficacy and reduces ascites in ovarian cancer models by normalizing the tumor stroma. Proc Natl Acad Sci U S A 116(6):2210-2219. doi:10.1073/pnas.1818357116

48. Chauhan VP, Martin JD, Liu H, Lacorre DA, Jain SR, Kozin SV et al (2013) Angiotensin inhibition enhances drug delivery and potentiates chemotherapy by decompressing tumour blood vessels. Nat Commun 4:2516. doi:10.1038/ncomms3516

49. Li M, Kroetz DL. Bevacizumab-induced hypertension: Clinical presentation and molecular understanding. 2018(1879-016X (Electronic))

50. Ong HT, Ong LM, Ho JJ (2013) Angiotensin-Converting Enzyme Inhibitors (ACEls) and AngiotensinReceptor Blockers (ARBs) in Patients at High Risk of Cardiovascular Events: A Meta-Analysis of 10 Randomised Placebo-Controlled Trials. ISRN Cardiol 2013:478597. doi:10.1155/2013/478597

\section{Figures}




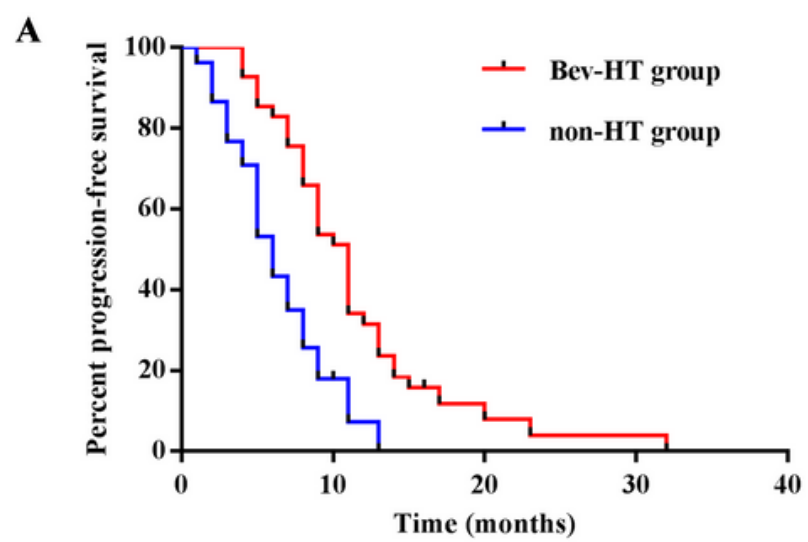

C

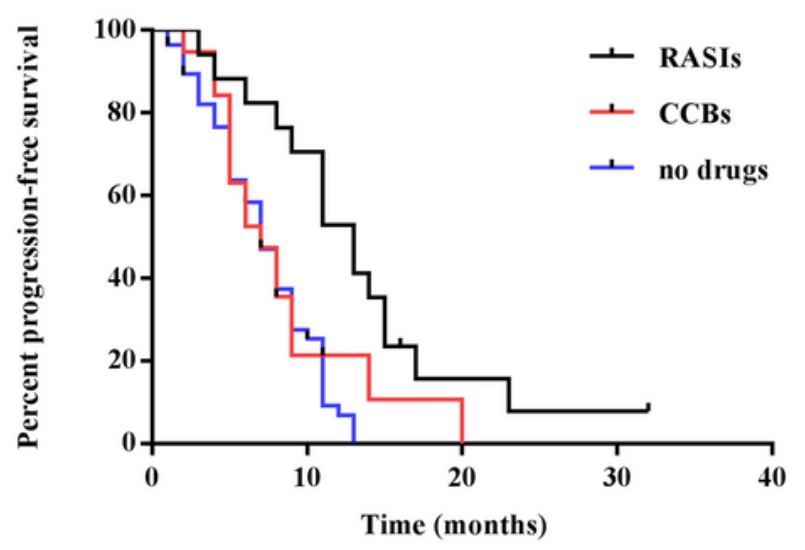

$\mathbf{E}$

Variable
Rercent progression-free survival
RASI use
Diabetes
Overall Survival
RASI use
$\begin{gathered}\text { Irinotecan-based regime } \\ \text { 7-12 Bev cycles }\end{gathered}$

B

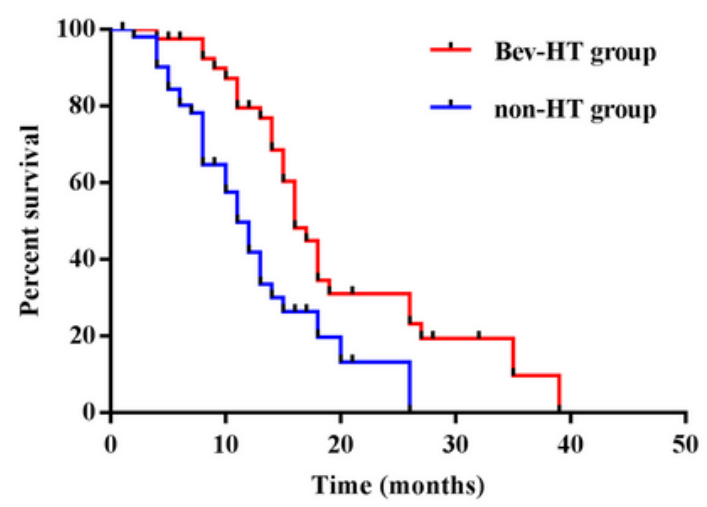

D

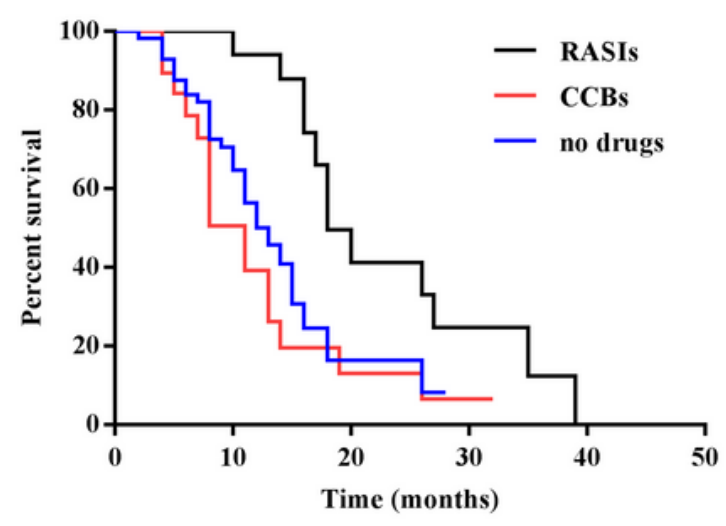

\section{Figure 1}

RASI adjunctive treatment improves survival in patients with mCRC. (A) PFS curves according to hypertensive status. (B) OS curves according to hypertensive status. (C) PFS curves according to antihypertensive treatment. (D) OS curves according to antihypertensive treatment. (E) The results of multivariate analysis of patient characteristics. HT, hypertension; RASIs, renin-angiotensin system 
inhibitors; CCBs, calcium channel blockers; SBP, systolic blood pressure; DBP, diastolic blood pressure; Bev, bevacizumab; $95 \% \mathrm{Cl}$, 95\% confidence interval.

A

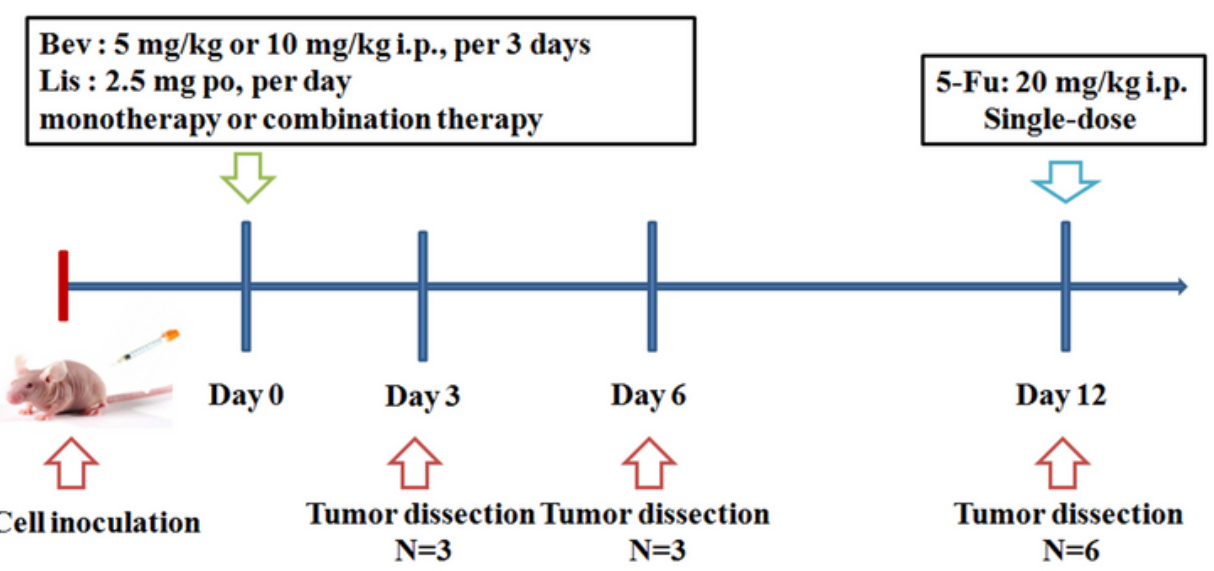

B

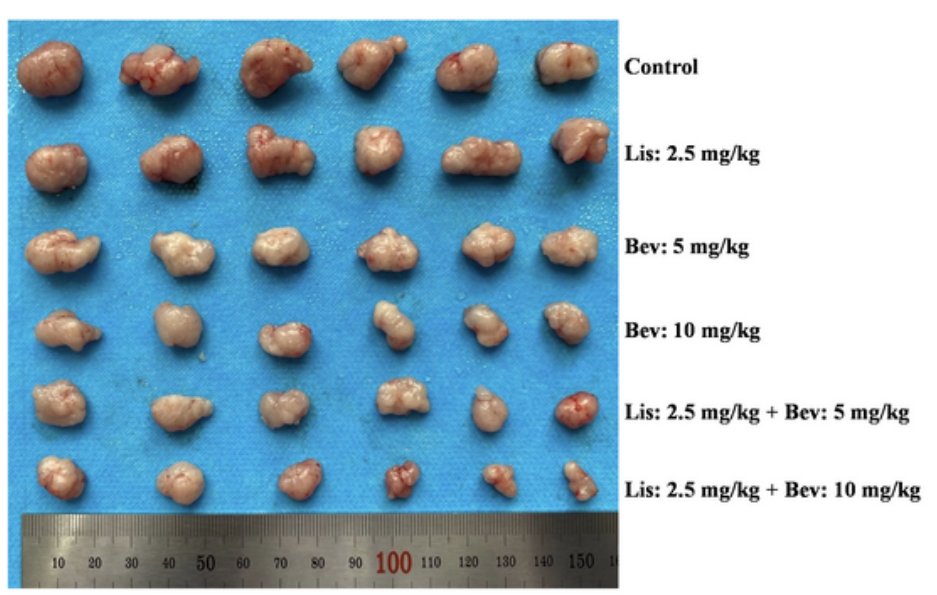

C

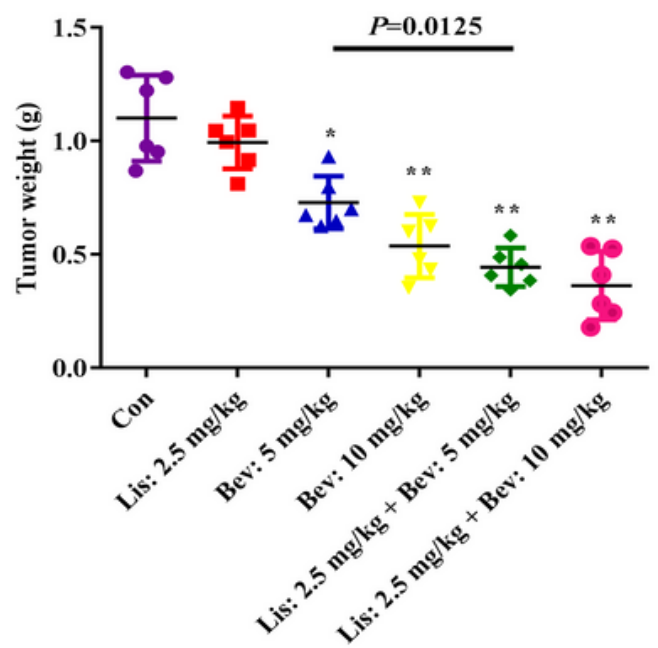

D

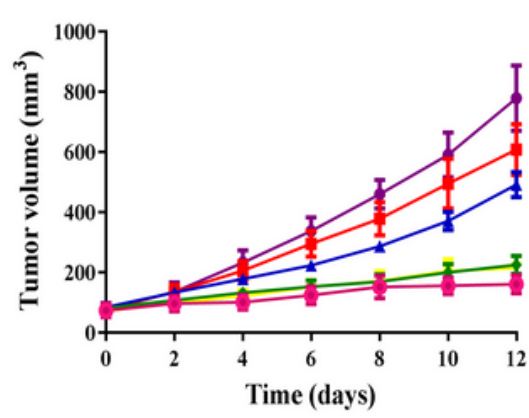

$\mathbf{E}$

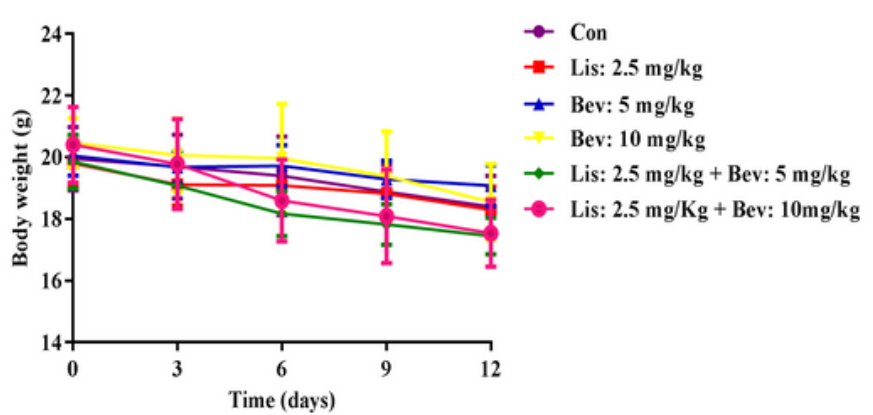

Figure 2

Bev and Lis synergistically inhibit tumor growth in HCT116 xenografts. (A) Schematic of the study design. BALB/c nude mice were subcutaneously injected in the bilateral flank with HCT116 cells. The mice were treated with Bev at $5 \mathrm{mg} / \mathrm{kg}$ or $10 \mathrm{mg} / \mathrm{kg}$ by intraperitoneal injection every 3 days and/or Lis at 
$2.5 \mathrm{mg} / \mathrm{kg}$ orally once daily. (B) Images of tumors excised from the mice $(\mathrm{n}=6)$. (C) Means of the tumor weights. (D) Tumor growth curves were generated by measuring tumor volumes every 2 days. (E) Means of the mouse weights. Data represent the mean \pm SEM. ${ }^{*} P<0.05,{ }^{*} P<0.01$ versus the control group. Con, control; Bev, bevacizumab; Lis, lisinopril; 5-Fu, 5-fluorouracil.

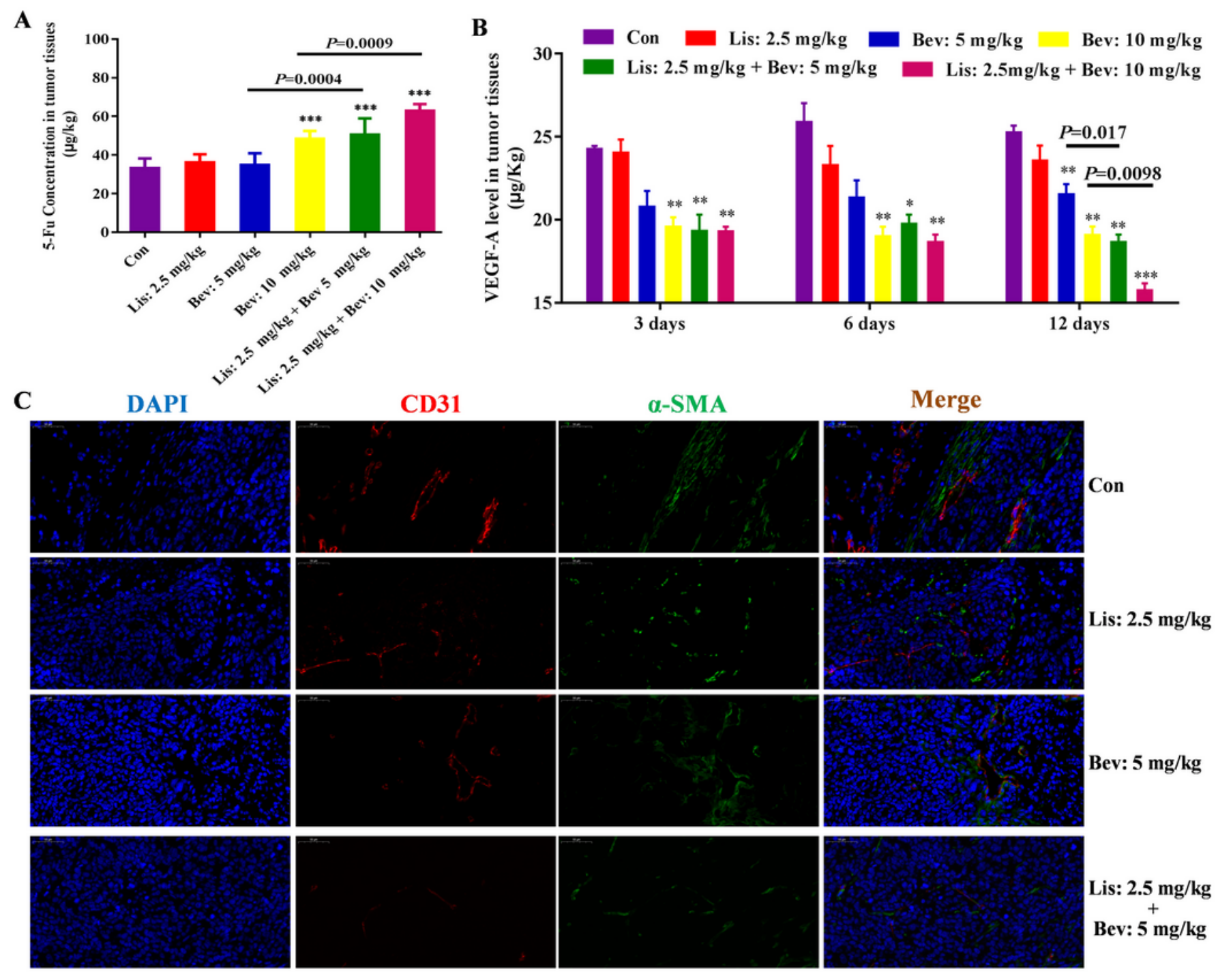

D

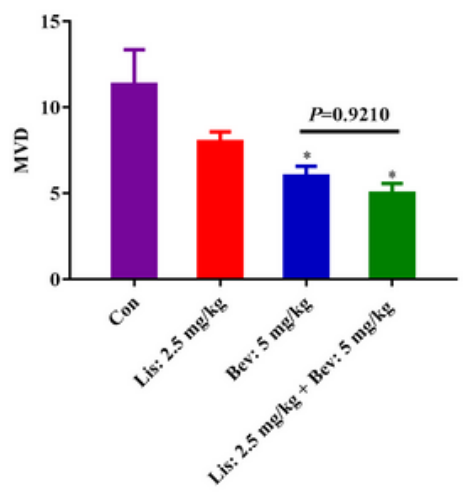

E

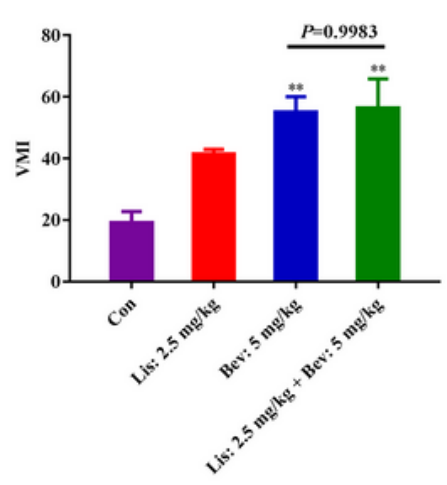

F

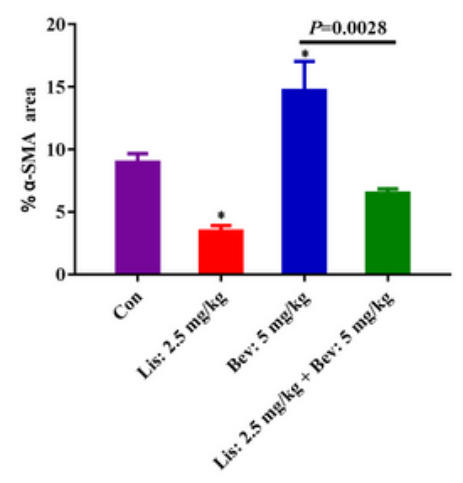

Figure 3 
Synergistic effects of Bev and Lis on 5-Fu tissue penetration and angiogenesis in HCT116 xenografts (A) Synergistic effect of Bev and Lis on 5-Fu concentration in tumor tissues $(n=6)$. (B) VEGFA levels in tumor tissues at 3,6 , and 12 days after Lis and Bev administration. (C) Immunofluorescence staining images of

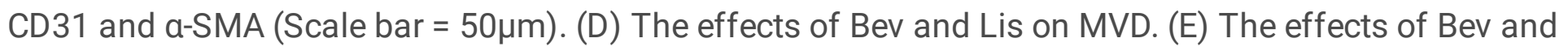
Lis on VMI. (F) The proportions of a-SMA-positive areas were quantified using ImageJ software. Data represent the mean \pm SEM. ${ }^{*} P<0.05, * * P<0.01, * \star \star P<0.001$ versus the control group. Con, control; Bev, bevacizumab; Lis, lisinopril; 5-Fu, 5-fluorouracil; VEGFA, vascular endothelial growth factor A; MVD, microvessel density; VMI, vascular maturity index. 
A
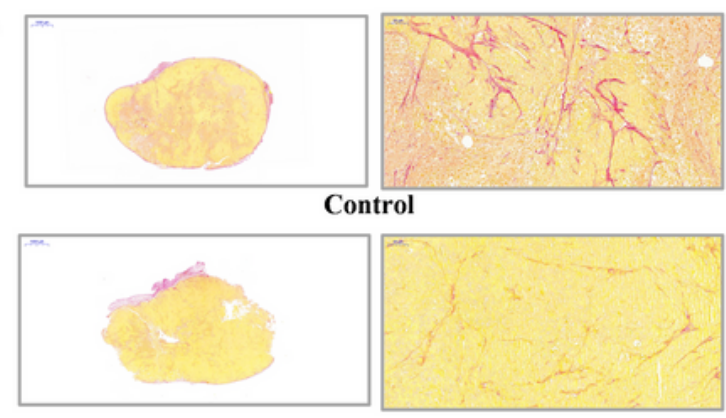

Lis $2.5 \mathrm{mg} / \mathrm{Kg}$

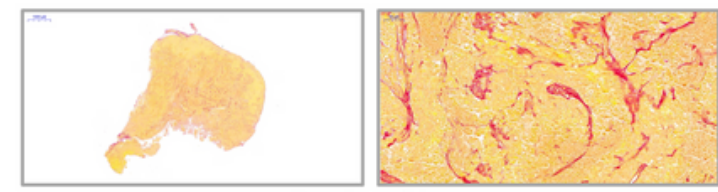

Bev $5 \mathrm{mg} / \mathrm{Kg}$

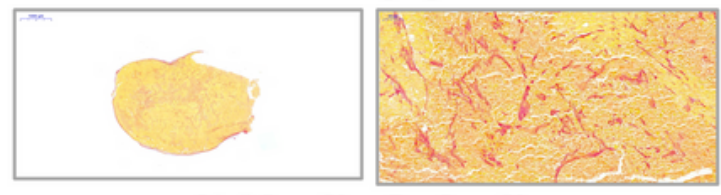

Lis $2.5 \mathrm{mg} / \mathrm{Kg}+\operatorname{Bev} 5 \mathrm{mg} / \mathrm{Kg}$

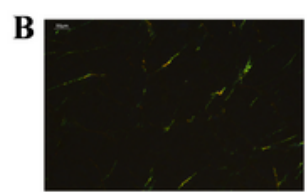

Control

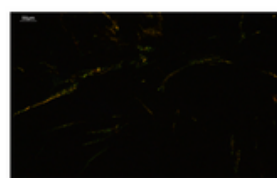

Lis $2.5 \mathrm{mg} / \mathrm{Kg}$

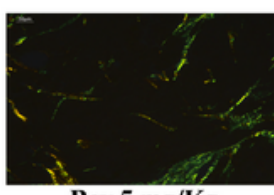

Bev $5 \mathrm{mg} / \mathrm{Kg}$

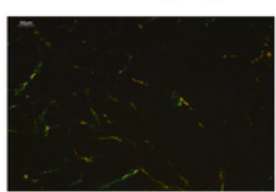

Lis $2.5 \mathrm{mg} / \mathrm{Kg}+$ Bev $5 \mathrm{mg} / \mathrm{Kg}$

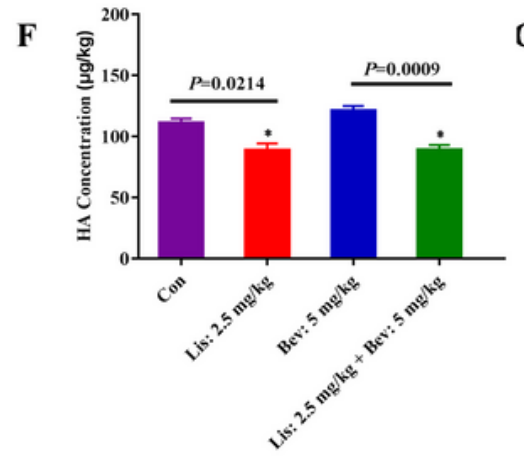

G

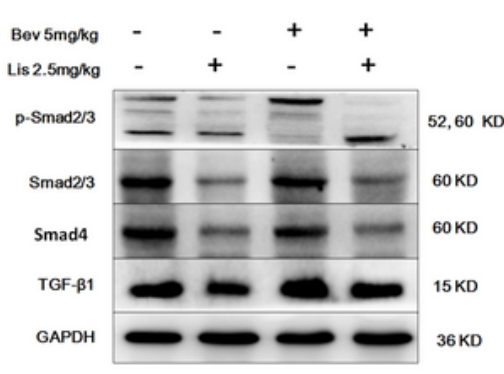

I

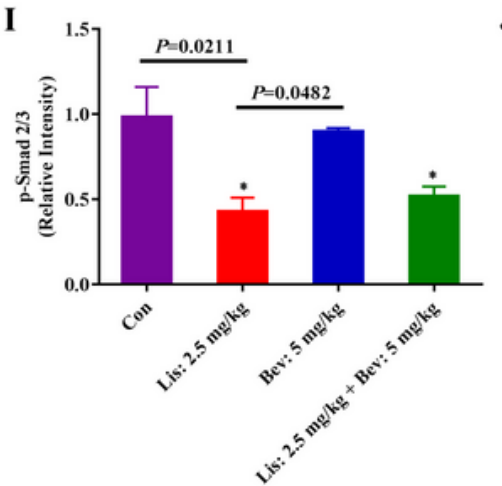

$\mathbf{J}$

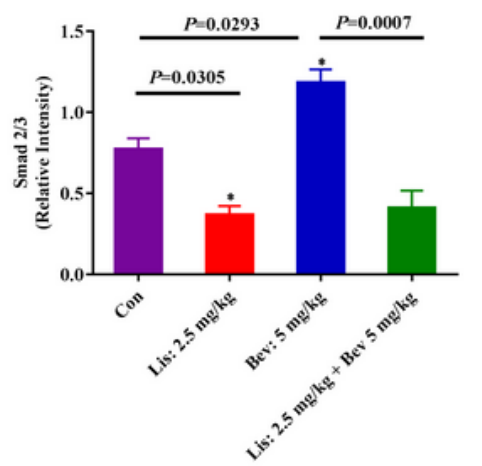

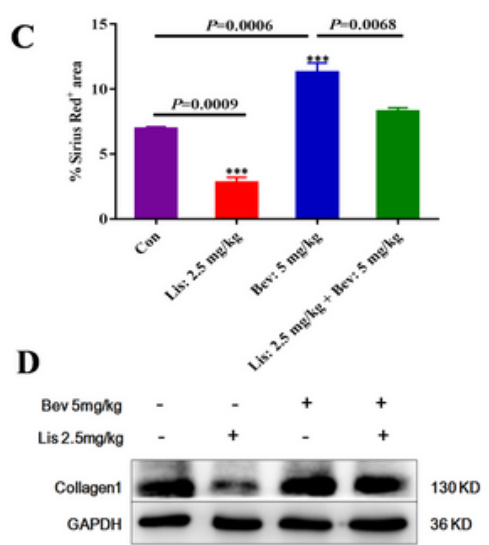

E

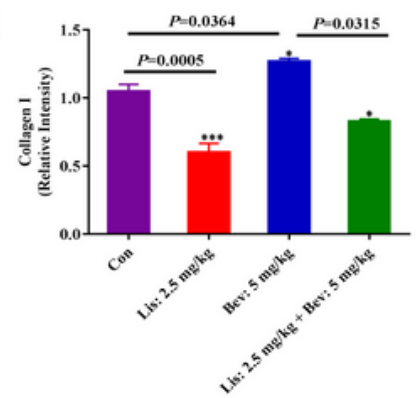

H

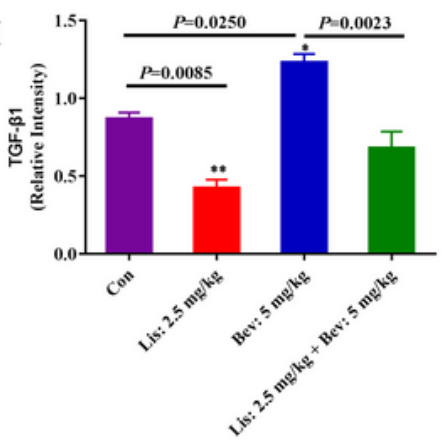

K

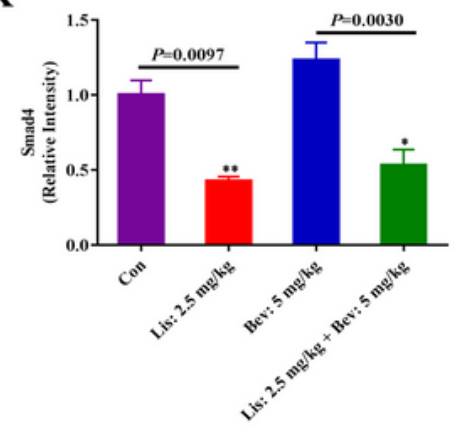

\section{Figure 4}

Lis reduced ECM remodeling by Bevin HCT116 xenograft models. (A) Collagen staining by Sirius Red and imaging by light microscopy (Scale bar $=1000 \mu \mathrm{m}$ and $50 \mu \mathrm{m}$ ). (B) Collagen staining by Sirius Red and imaging by polarized light (Scale bar $=50 \mu \mathrm{m})$. (C) The proportions of Sirius Red-positive areas were quantified using Image $J$ software. For each staining experiment, with three sections per tumor $(n=3)$. (D) Western blot analysis of collagen I in tumor tissues. (E) Quantitative collagen I protein expression ( $\mathrm{n}=$ 
3). (F) ELISA of the HA expression level $(n=6)$. (G) Western blot analysis of the protein expression of ECM markers in tumor tissues. $(\mathrm{H}-\mathrm{K})$ Quantitative protein expression levels. Data are the mean $\pm \mathrm{SEM}$. ${ }^{*} \mathrm{P}<0.05,{ }^{*} \mathrm{P}<0.01,{ }^{\star} * * \mathrm{P}<0.001$ versus the control group. ECM, extracellular matrix; Con, control; Bev, bevacizumab; Lis, lisinopril; HA, hyaluronic acid; TGF- $\beta 1$,transforming growth factor $\beta 1$.

\section{Supplementary Files}

This is a list of supplementary files associated with this preprint. Click to download.

- supplimentarymaterialRTSJH20210806.docx 